\title{
Biochar, soil fertility, and environment
}

\author{
Pellegrino Conte
}

Published online: 15 October 2014

(C) Springer-Verlag Berlin Heidelberg 2014

Biochar is a carbonaceous material obtained by pyrolysis of biomass feedstock. It is deliberately applied to soils in order to improve fertility and mitigate greenhouse gas emissions. In fact, on one hand, biochar changes physical-chemical soil properties, thereby affecting soil fertility. On the other hand, biochar is resistant to chemical and biochemical degradation. Therefore, its use allows carbon sequestration in soils and consequent reduction of carbon dioxide to the atmosphere. Moreover, biochar is a porous material showing good sorption properties for inorganic and organic pollutants. Because of this, biochar can be applied for environmental remediation.

Notwithstanding the positive effects in environmental applications, many studies also report about adverse issues deriving from biochar uses. In fact, controversial results concerning use of biochar as amending material can be found in the literature. Due to the direct relationship between structural/molecular characteristics and chemical/ biochemical properties, it must be pointed out that a deep characterization either by innovative physical-chemical techniques or by traditional chemical investigations is of paramount importance in order to reveal biochar role in environmental compartments, to address its agronomical and environmental uses and to allow meaningful pre-application quality assessments.

In the present special issue, a number of papers are presented in order to evaluate the role of biochar in removing heavy metals from contaminated water, in reducing greenhouse gas emissions from arable soils, in considering its role in soil fertility through comparison of its nature to those of humic substances, in affecting root length, and in improving nitrification rate in biochar-amended soils.
P. Conte $(\bowtie)$

Dipartimento di Scienze Agrarie e Forestali, Università degli Studi di

Palermo, v.le delle Scienze ed. 4, 90128 Palermo, Italy

e-mail: pellegrino.conte@unipa.it 